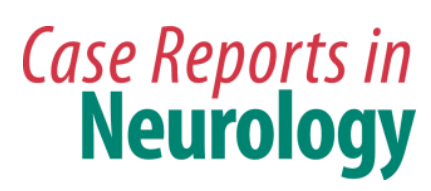

\title{
Agraphia Caused by Left Thalamic Hemorrhage
}

\author{
Aiko Osawa $^{a}$ Shinichiro Maeshima $^{a}$ Fumitaka Yamane $^{b}$ \\ Nahoko Uemiya $^{\mathrm{b}}$ Ikuo Ochiai ${ }^{\mathrm{b}}$ Tomoyuki Yoshihara $^{\mathrm{b}}$ \\ Shoichiro Ishihara ${ }^{b}$ Norio Tanahashic \\ Departments of ${ }^{a}$ Rehabilitation Medicine, ${ }^{b}$ Endovascular Neurosurgery and ${ }^{c}$ Neurology, \\ Saitama Medical University International Medical Center, Hidaka, Japan
}

\section{Key Words}

Cerebral hemorrhage $\cdot$ Agraphia $\cdot$ Aphasia $\cdot$ Stroke

\begin{abstract}
A 71-year-old right-handed man was admitted to our hospital with right hemiparesis and sensory impairment associated with mild aphasia. Although aphasia gradually resolved within 2 weeks after stroke onset, his writing ability remained disturbed. A computed tomography (CT) scan at stroke onset revealed a hematoma in the left thalamus, but no cortical lesions were observed. Further, a single-photon emission CT (SPECT) scan showed decreased blood flow in the left thalamus, in the cortical region extending from the left superior temporal gyrus to the parietal lobe, and in the frontal lobe. It is possible that agraphia may have directly resulted from the thalamic lesion, but SPECT findings strongly suggested that a general decrease in left cortical function concomitant with a disruption of the thalamocortical and cortico-thalamocortical projection fibers produced these cognitive deficits.
\end{abstract}

\section{Introduction}

Functional involvement of the thalamus in cognitive processing was first proposed by Hillemand [1] and Lhermitte [2], who described atypical aphasic symptoms after pathologically confirmed left thalamic hemorrhage. These anecdotal descriptions were later confirmed by Fisher [3], who reported aphasia, agnosia, and sensory deficits as the main clinical features of left thalamic hemorrhage. It is now accepted that left thalamic hemorrhage often causes aphasia characterized by fluent but sometimes hypophonic speech, paraphasia, and moderate to mild disturbances in auditory and reading comprehension [4]. 
Osawa et al.: Agraphia Caused by Left Thalamic Hemorrhage

Agraphia, an acquired impairment in writing ability with preserved oral language competence and reading [5], is often observed with other aphasic symptoms after stroke. Enduring agraphia after recovery from aphasia has also been reported in stroke patients, but few such cases have been described following thalamic hemorrhage [6]. Here we report a case of sustained agraphia with transient aphasia and agnosia following left thalamic hemorrhage.

\section{Case Report}

The patient was a 71-year-old right-handed man with 12 years of formal education. He had no history of diabetes, hypertension, or neurological or metabolic complications, and he had no preexisting neuropsychological deficits. The patient initially experienced weakness of the right arm and leg and had difficulty in walking. He was transported to our hospital by ambulance, where he was diagnosed with cerebral hemorrhage and received conservative treatment. He came to our department the following day for rehabilitation.

On admission, he was alert but disoriented with regard to time and place because of mild aphasia. He also presented with right hemiplegia, impaired thermal nociception, and impaired deep sensation on his right side. His visual acuity and visual field were normal, and there were no other detectable abnormalities of the central nervous system.

\section{Neuropsychological Findings}

Formal language assessment was undertaken 3 days after stroke onset using the Standard Language Test for Aphasia (SLTA; fig. 1). He had fluent spontaneous speech with only occasional difficulty in choosing the correct word (paraphasia), and he could repeat spoken words. He had slight difficulty in visual recognition of words and aural comprehension at the sentence level, but he could read words aloud, whether written in Kanji (Chinese-based characters) or Kana (syllable-based characters). However, he had difficulty in writing both types of characters. He scored 16/30 on the Mini-Mental State Examination (MMSE), with poor performance in orientation, attention and calculation, and reproduction. His Raven's Colored Progressive Matrices (RCPM) score was 18/36 and that on the Frontal Assessment Battery (FAB) was 11/18. A word fluency test requiring the patient to produce exemplars from given categories resulted in scores of 17, 7, and 9 for the categories 'animal', 'fruit', and 'vehicle', respectively. A test requiring the production of words that begin with specified letters resulted in scores of 0, 6, and 7 for the letters 'shi', 'i', and 're', respectively.

\section{Neuroradiological Findings}

A head computed tomography (CT) scan at stroke onset revealed a thalamic hemorrhage in the left lateral and posterior thalamus with slight extension to the posterior limb of the internal capsule (fig. 2a). Single-photon emission CT (SPECT) performed around the same time showed decreased blood flow in the left thalamus, in the left cortex extending from the superior temporal gyrus to the parietal lobe, and in the frontal lobe (fig. 2b).

\section{Clinical Course}

Aphasia gradually resolved as the patient's SLTA score improved to population norms in the areas of comprehension and oral language by 2 weeks after stroke onset. His right hemiplegia improved gradually but remained mildly hemiparetic. The patient could manage to write with his right hand, but used his left hand for writing because of his difficulty in delicate motor control. Despite signs of general cognitive recovery, his writing ability 
Osawa et al.: Agraphia Caused by Left Thalamic Hemorrhage

remained clearly disturbed. Most errors were incorrect responses to word dictation, termed paragraphia, although the selection and array of characters used were normal. He could transcribe characters and generally wrote the characters in the correct stroke order, but he often substituted one character for another with a similar shape or sound, perseverated, and wrote characters with $180^{\circ}$ rotation (mirror script) (fig. 3). He could write 67 of 102 Kana characters and 62 of 160 Kanji characters, which are learned during the second grade in Japan. At 2 weeks after stroke onset, Wechsler Adult Intelligence Scale-III (WAIS-III) scores were 83 for Verbal Intelligence Quotient (IQ), 65 for Performance IQ, and 72 for Full Scale IQ while the Wechsler Memory Scale-Revised (WMS-R) scores were 77 for Verbal Memory, 73 for Visual Memory, 74 for General Memory, 81 for Attention and Concentration, and 57 for Delayed Recall. Compared with the results obtained 3 days after stroke onset, the scores were slightly higher on the MMSE (20/30 vs. $16 / 30)$, RCPM (26/36 vs. $18 / 36)$, and FAB $(12 / 18$ vs. 11/18). In contrast, word fluency test results were similar for both categories $(16$, 8 , and 9) and specific first letters $(7,4$, and 6), although the 'shi' deficit had recovered. At 6 weeks after stroke onset, his writing deficit remained despite improvement in other language abilities (fig. 1).

\section{Discussion}

In our patient, oral language and reading comprehension rapidly improved over the first 2 weeks after stroke onset, but agraphia continued for at least 6 weeks. He could transcribe Kanji and Kana characters and write them with the correct stroke order, and the selection and array of characters were normal, but there were mistakes in transcription. The act of writing engages multiple cortical areas; thus, agraphia may result from a general reduction in brain function or acute delirium, decreased memory retention due to thalamic lesions, or secondary cortical impairments [7]. However, in the present case, the patient exhibited no disturbance of consciousness and had relatively intact general cognitive function at 2 weeks after stroke onset, while agraphia for Kana or Kanji characters remained.

The Japanese language has two writing systems, Kanji and Kana, which are often used for comparison of handwriting [8]. Some patients with left posterior inferior temporal lobe lesions may have agraphia specifically for Kanji $[9,10]$, while some patients with frontal or parietal lobe lesions are unable to write Kanji or Kana. Aiba et al. [6] reported a case of agraphia following thalamic hemorrhage in which the degree of agraphia for Kanji was greater than that for Kana. In our patient, a SPECT scan revealed decreased blood flow over an expansive area of the left frontal and temporal lobes. Maeshima et al. [11] reported a case of thalamic hemorrhage causing agraphia for Kanji and mentioned that a SPECT scan showed decreased blood flow in the left thalamus, left superior temporal gyrus extending to the parietal lobe, and the frontal lobe. Sakurai et al. [12] suggested that two separate lesions of the left thalamus could cause agraphia; damage to the ventral lateral nucleus can impair Kana character recall, whereas damage to the dorsomedial nucleus can cause a deficit primarily for Kanji character recall. In our patient, the hematoma extended throughout both the lateral and medial left thalamus, suggesting a possible explanation for agraphia for both Kanji and Kana characters. A CT scan of the head revealed no abnormalities, except for those in the left thalamus, but SPECT showed a decrease in regional cerebral blood flow in the left temporal and parietal lobes and in the frontal lobe (as well as in the thalamus). It is well known that neuropsychological disturbances in patients with posterolateral thalamic hemorrhage are prominent, with aphasia in those with left-sided lesions and hemineglect in those with right-sided lesions [13, 14]. Crosson [15] formulated the most extensive 
theoretical model for the roles of different subcortical structures in language comprehension and production. According to this model, the striatum and cortico-striato-pallidothalamocortical loop are as important as anterior and posterior language structures (Broca's area and Wernicke's area), with striatal and thalamic areas particularly important for correcting errors in meaning. The dorsomedial nucleus of the thalamic medial nuclear group projects mainly to the prefrontal area, the ventral anterior nucleus of the ventral nuclear group to the premotor area, the posterior lateral nucleus and pulvinar nucleus of the lateral nuclear group to the parietal lobe, the medial geniculate body to the primary visual area of the occipital lobe, and the medial geniculate body to the primary auditory area of the temporal lobe [15]. Aphasia and neglect were likely caused by damage to the thalamocortical and cortico-thalamocortical projection fibers due to thalamic hemorrhage, resulting in a widespread decrease in cortical function $[16,17]$. We suggested that agraphia in our patient may be caused by a general decrease in left cortical function concomitant with a disruption of the thalamocortical and cortico-thalamocortical projection fibers resulting from thalamic hemorrhage in the lateral nuclear group.

\section{References}

1 Hillemand P: Contribution à l'étude des syndromes thalamiques; thèse, Paris, 1925.

2 Lhermitte J: Symptomatologie de l'hémorragie du thalamus. Rev Neurol (Paris) 1936;65:89-93.

-3 Fisher CM: The pathologic and clinical aspects of thalamic hemorrhage. Trans Am Neurol Assoc 1959;84:56-59.

4 Alexander MP, LoVerme SR Jr: Aphasia after left hemispheric intracerebral hemorrhage. Neurology 1980;30:1193-1202.

5 Toyokura M, Kobayashi R, Aono KA: A case of pure agraphia due to left thalamic hemorrhage. Tokai J Exp Clin Med 2010;35:89-94.

6 Aiba E, Souma Y, Aiba T, Kulita I, Kishida K: Two cases of pure agraphia developed after thalamic hemorrhage. No To Shinkei 1991;43:275-281.

7 Chedru F, Geschwind N: Writing disturbances in acute confusional state. Neuropsychologia 1972;10:343353.

8 Iwata M: Kanji versus Kana: neuropsychological correlates of the Japanese writing system. Trends Neurosci 1984;7:290-293.

-9 Sakurai Y, Matsumura K, Iwatsubo T, Momose T: Frontal pure agraphia for kanji or kana: dissociation between morphology and phonology. Neurology 1997;49:946-952.

10 Maeshima S, Ueyoshi A, Matsumoto T, Okita R, Yamaga H, Ozaki F, Moriwaki H, Roger P: Agraphia in Kanji after a contusional haemorrhage in the left temporo-occipital lobe. J Neurol Neurosurg Psychiatry 2002;72:126-127.

-11 Maeshima S, Osawa A, Ogura J, Sugiyama T, Kurita H, Satoh A, Tanahashi N: Functional dissociation between Kana and Kanji: agraphia following a thalamic hemorrhage. Neurol Sci 2011;33:409-413.

-12 Sakurai Y, Yoshida Y, Sato K, Sugimoto I, Mannen T: Isolated thalamic agraphia with impaired grapheme formation and micrographia. J Neurol 2011;258:1528-1537.

13 Kumral E, Kocaer T, Ertübey NÖ, Kumral K: Thalamic hemorrhage. A prospective study of 100 patients. Stroke 1995;26:964-970.

14 Kawahara N, Sato K, Muraki M, Tanaka K, Kaneko M, Uemura K: CT classification of small thalamic hemorrhages and their clinical implications. Neurology 1986;36:165-172.

15 Crosson B: Role of the dominant thalamus in language: a review. Psychol Bull 1984;96:491-517.

16 Perani D, Vallar G, Cappa S, Messa C, Fazio F: Aphasia and neglect after subcortical stroke. A clinical/cerebral perfusion correlation study. Brain 1987;110:1211-1229.

$\checkmark 17$ Vallar G, Perani D, Cappa SF, Messa C, Lenzi GL, Fazio F: Recovery from aphasia and neglect after subcortical stroke: neuropsychological and cerebral perfusion study. J Neurol Neurosurg Psychiatry 1988;51:12691276. 
-3 days after the onset -2 weeks after the onset -6 weeks after the onset

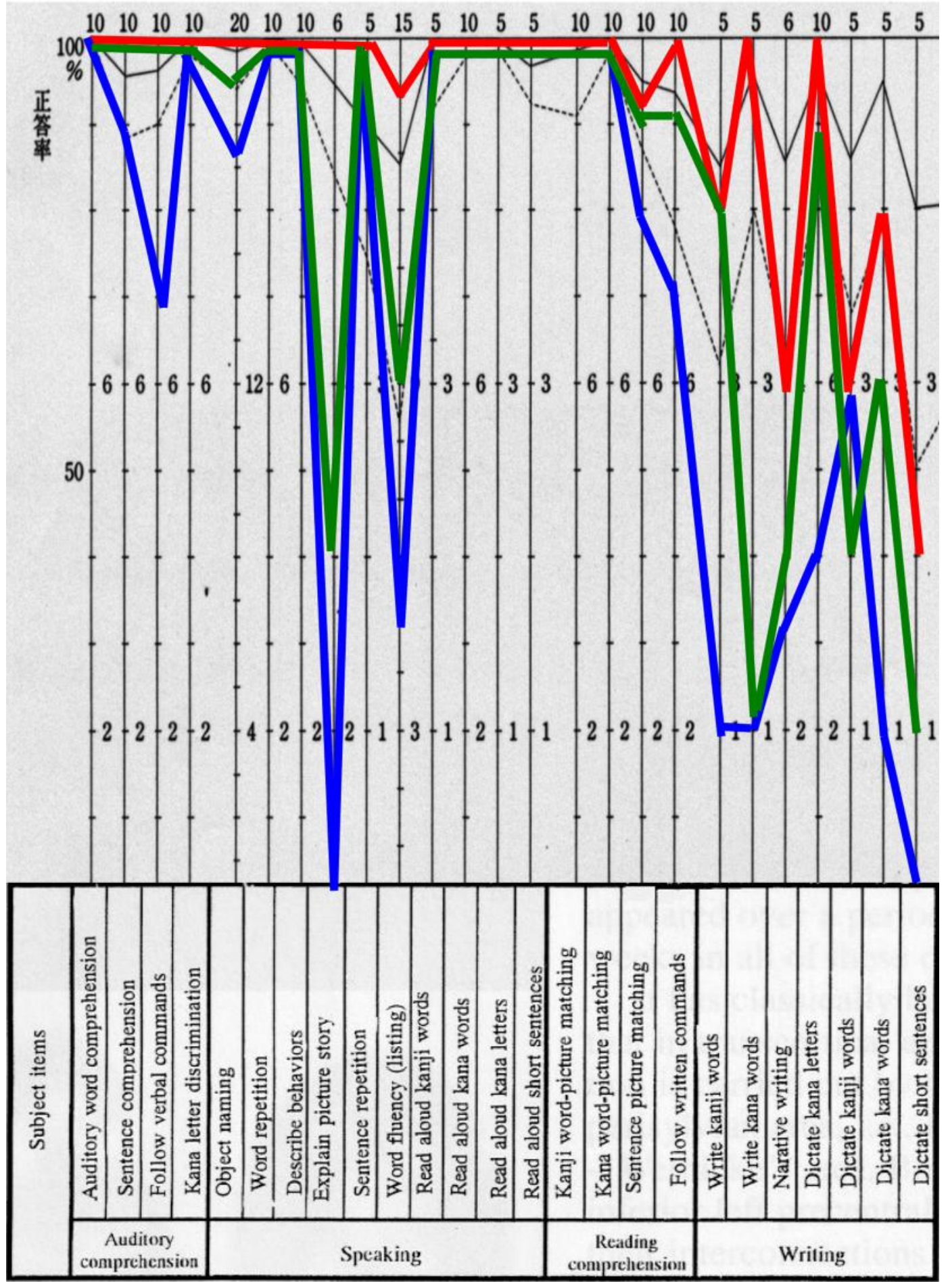

Fig. 1. Formal language assessment was undertaken using the SLTA. 


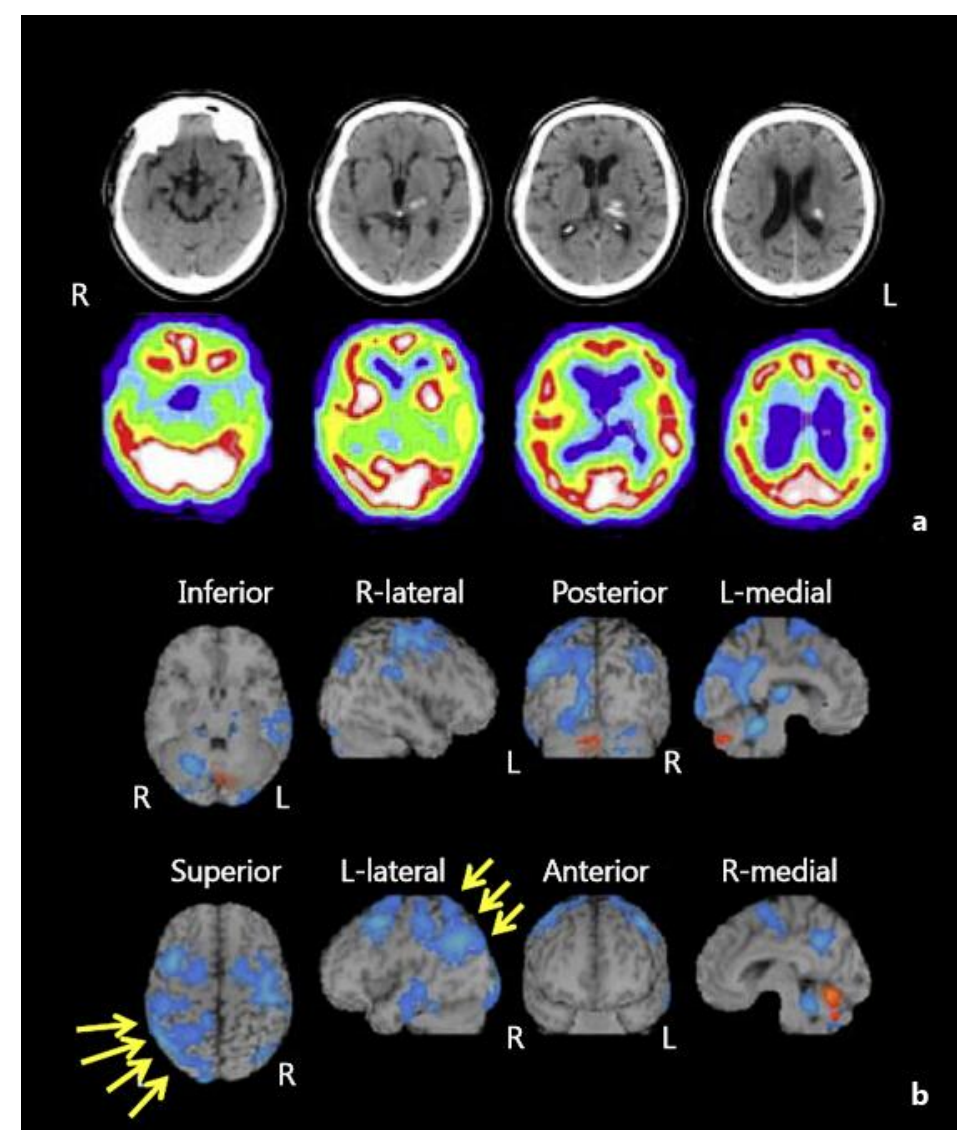

Fig. 2. a A head CT scan at stroke onset revealed a hematoma expanding from inside the posterior left thalamus. b SPECT performed around the same time showed decreased blood flow in the left thalamus, in the left cortex extending from the superior temporal gyrus to the parietal lobe, and in the frontal lobe. 


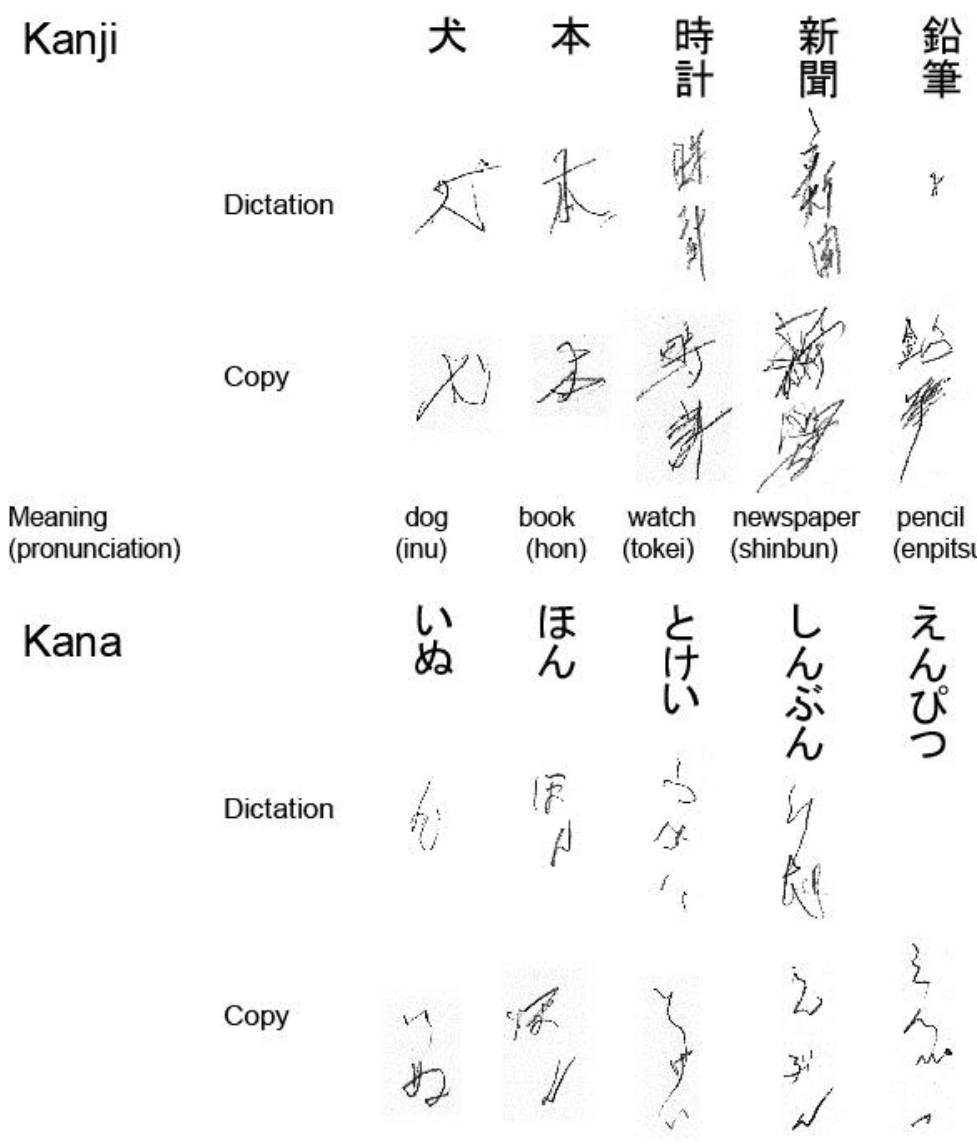

Fig. 3. Most errors were incorrect responses to word dictation, termed paragraphia. The patient could transcribe characters and generally wrote the characters in the correct stroke order, but he often substituted one character for another with a similar shape or sound, perseverated, and wrote characters with $180^{\circ}$ rotation (mirror script). 